\title{
OPEN Biological age and lifestyle in the diagnosis of metabolic syndrome: the NHIS health screening data, 2014-2015
}

\author{
Chul-Young Bae ${ }^{1}$, Meihua Piao ${ }^{2}$, Miyoung Kim ${ }^{1}$, Yoori $\mathrm{Im}^{1}$, Sungkweon Kim¹, Donguk Kim³ \\ Junho $\mathrm{Choi}^{4} \&$ Kyung Hee Cho ${ }^{5}$
}

Metabolic syndrome (MS) is diagnosed using absolute criteria that do not consider age and sex, but most studies have shown that the prevalence of MS increases with age in both sexes. Thus, the evaluation of MS should consider sex and age. We aimed to develop a new index that considers the age and sex for evaluating an individual's relative overall MS status. Data of $16,518,532$ subjects $(8,671,838$ males and $7,846,694$ females) who completed a validated health survey of the National Health Insurance Service of the Republic of Korea (2014-2015) were analyzed to develop an MS-biological age model. Principal component score analysis using waist circumference, pulse pressure, fasting blood sugar, triglyceride levels, and high-density lipoprotein level, but not age, as independent variables were performed to derive an index of health status and biological age. In both sexes, the age according to the MS-biological age model increased with rising smoking and alcohol consumption habits and decreased with rising physical activity. Particularly, smoking and drinking affected females, whereas physical activity affected males. The MS-biological age model can be a supplementary tool for evaluating and managing MS, quantitatively measuring the effect of lifestyle changes on MS, and motivating patients to maintain a healthy lifestyle.

Metabolic syndrome (MS) is a global health problem involving a cluster of several cardiovascular risk factors ${ }^{1}$. The incidence of MS has been increasing worldwide, including in South Korea ${ }^{2}$. The Korea National Health and Nutrition Examination Survey reports that the prevalence of MS, assessed according to the modified National Cholesterol Education Program-Adult Treatment Panel III (NCEP-ATP III) criteria, has increased from $24.9 \%$ in 1998 to $34.7 \%$ in $2012^{3}$. MS includes various combinations of insulin resistance, abdominal adiposity, dyslipidemia, and/or hypertension, and its prevalence increases with age. Moreover, MS is associated with a high risk of morbidity and mortality owing to cardiovascular disease (CVD) and type 2 diabetes ${ }^{4}$.

There is currently no standard definition for MS, causing confusion regarding whether they identify the same individuals or represent risk factor surrogates ${ }^{5}$. The NCEP-ATP III definition is the easiest to apply clinically and epidemiologically because it uses straightforward criteria that can be readily measured ${ }^{6}$. However, although metabolic risk factor clustering is consistent, the categorical diagnosis of MS is variable during adolescence ${ }^{7}$. Moreover, the conventional diagnosis of MS utilizes absolute criteria that does not consider the subject's age and sex despite most studies showing that the prevalence of MS increases with age in both men and women ${ }^{8-12}$. Especially in women, the prevalence rapidly increases after menopause. Menopause is associated with the redistribution of body weight and weight gain in most women, and weight gain and obesity largely underlie the increased prevalence of MS in postmenopausal women ${ }^{13}$. This rise in prevalence is known to be due to the increase of chronic diseases such as central obesity, hypertension, diabetes, dyslipidemia, and deficiency in sex hormones with increasing age ${ }^{14}$.

Variables that show certain patterns according to age are called biomarkers of aging ${ }^{15}$. For example, waist circumstance is known as the most reliable biomarker by which we can diagnose central obesity and predict visceral fat amount, which increases with age ${ }^{16}$. Biological age (BA) estimated by the biomarkers of aging may

\footnotetext{
${ }^{1}$ MediAge Research Center, Sungnam, Republic of Korea. ${ }^{2}$ Office of Hospital Information, Seoul National University Hospital, 101 Daehak-ro, Jongno-gu, Seoul 03080, Republic of Korea. ${ }^{3}$ Department of Big Data, National Health Insurance Corporation, Wonju, Republic of Korea. ${ }^{4}$ Department of Family Medicine, National Health Insurance Service Ilsan Hospital, Goyang, Republic of Korea. ${ }^{5}$ Research and Analysis Team, National Health Insurance Service Ilsan Hospital, Goyang, Republic of Korea. ${ }^{\circledR}$ email: parkmihua@snu.ac.kr
} 


\begin{tabular}{|c|c|c|c|c|}
\hline \multirow[b]{2}{*}{ Parameters } & \multirow[b]{2}{*}{ Inclusion Criteria } & \multicolumn{3}{|l|}{ Mean \pm SD } \\
\hline & & Total $(n=16,518,532)$ & Male $(n=8,671,838)$ & Female $(n=7,846,694)$ \\
\hline Age, years & $>20$ & $50.33 \pm 14.3$ & $48.78 \pm 14.16$ & $52.04 \pm 14.26$ \\
\hline Waist circumference, $\mathrm{cm}$ & $60-105$ & $80.86 \pm 8.84$ & $84.17 \pm 7.63$ & $77.2 \pm 8.63$ \\
\hline \multicolumn{5}{|l|}{ Blood pressure (mmHg) } \\
\hline Systolic & $80-160$ & $121.58 \pm 13.45$ & $123.57 \pm 12.45$ & $119.37 \pm 14.16$ \\
\hline Diastolic & $50-100$ & $75.55 \pm 9.15$ & $77.1 \pm 8.78$ & $73.82 \pm 9.24$ \\
\hline Mean arterial blood pressure & & $90.89 \pm 9.83$ & $92.59 \pm 9.23$ & $89.01 \pm 10.12$ \\
\hline Pulse pressure & & $46.03 \pm 9.38$ & $46.47 \pm 8.96$ & $45.55 \pm 9.80$ \\
\hline Fasting blood sugar $(\mathrm{mmol} / \mathrm{L})$ & $2.78-7.77$ & $5.33 \pm 0.71$ & $5.40 \pm 0.72$ & $5.25 \pm 0.68$ \\
\hline Triglyceride (mmol/L) & $0.57-4.52$ & $1.42 \pm 0.73$ & $1.56 \pm 0.79$ & $1.27 \pm 0.63$ \\
\hline High-density lipoprotein (mmol/L) & $0.52-2.33$ & $1.40 \pm 0.32$ & $1.33 \pm 0.30$ & $1.49 \pm 0.32$ \\
\hline
\end{tabular}

Table 1. General characteristics of the participants. Mean arterial blood pressure $=$ (systolic blood pressure $+2 \times$ diastolic blood pressure $) / 3$; Pulse pressure $=($ systolic blood pressure - diastolic blood pressure $)$. SI conversion factors: to convert fasting blood sugar to $\mathrm{mmol} / \mathrm{L}$, multiply by 0.0555 ; triglyceride to $\mathrm{mmol} / \mathrm{L}$, multiply by 0.0113 ; high-density lipoprotein to $\mathrm{mmol} / \mathrm{L}$, multiply by 0.0259 . SD standard deviation.

\begin{tabular}{|l|c|c|c|c|}
\hline & \multicolumn{2}{|l|}{$\begin{array}{l}\text { Pearson } \\
\text { correlation } \\
\text { coefficients } \\
\text { between age and } \\
\text { parameters }\end{array}$} & \multicolumn{2}{l|}{$\begin{array}{l}\text { Standardized scoring } \\
\text { coefficients }\end{array}$} \\
\cline { 2 - 5 } Parameters & Male & Female & Male & Female \\
\hline Waist circumference, cm & 0.092 & 0.356 & 0.42286 & 0.3722 \\
\hline Pulse pressure, mmHg & 0.190 & 0.354 & 0.13926 & 0.25941 \\
\hline Fasting blood sugar, mmol/L & 0.231 & 0.265 & 0.24737 & 0.28312 \\
\hline Triglyceride, mmol/L & -0.020 & 0.238 & 0.43813 & 0.37087 \\
\hline High-density lipoprotein, mmol/L & -0.037 & -0.221 & -0.40399 & -0.33814 \\
\hline
\end{tabular}

Table 2. The parameters influencing metabolic syndrome BA. PP (Pulse pressure) $=$ systolic blood pressure - diastolic blood pressure. All parameters are $P<0.001$.

serve as an indicator of identifying individuals at risk for age-related disorders, serving as a measure of relative fitness, and predicting disability in later life and mortality independent of chronological age ${ }^{17}$. Thus, MS diagnosis should consider biological age to overall evaluate and manage the health status and aging state in MS.

MS and its components, including dyslipidemia and hypertension, have been demonstrated to be common precursors of the development of type 2 diabetes and CVD and are risk factors for all-cause mortality ${ }^{18}$. Further, the risk of MS is known to be modified by diet, physical activity, smoking, drinking, and stress ${ }^{19,20}$. MS is associated with obesity and a sedentary lifestyle, both of which are modifiable, and thus, Misra et al. underlined the need for further efforts to promote a healthy lifestyle with increased physical activity to reduce obesity and the risk of $\mathrm{MS}^{21}$. Furthermore, individuals with MS should be identified at the early period therefore to attenuate their cardiovascular risk factors ${ }^{22}$. However, few studies have investigated approaches to quantify the effects of smoking, drinking, and physical activity in the risk of MS.

This study aimed to develop a new index that considers the age and sex for evaluating an individual's relative overall MS status and to quantitatively assess the association between lifestyle factors, including alcohol drinking, smoking, and physical activity, and MS-BA. Towards this goal, we created an MS-biological age (MS-BA) model using data from the National Health Insurance Service (NHIS) of the Republic of Korea.

\section{Results}

General characteristics. In total, $16,518,532$ subjects with an average age of $50.33 \pm 14.3$ years were evaluated. Of them, 8,671,838 were male (average age, $48.78 \pm 14.16$ years) and 7,846,694 were female (average age, $52.04 \pm 14.26$ years). The clinical parameters associated with MS are summarized in Table 1 .

MS-BA model. Correlation analysis and assessment of redundancy. The linear correlation between the diagnostic parameters of MS and age were analyzed. All parameters, except levels of triglycerides (TGs) and highdensity lipoprotein cholesterol (HDL-C) in males and levels of HDL-C in females, were positively correlated with age (Table 2). The mean arterial blood pressure (MBP) and pulse pressure (PP) could be calculated based on systolic blood pressure (SBP) and diastolic blood pressure (DBP) and could have strong correlations with each other. Thus, we chose the parameter with the highest correlation with age as the blood pressure indicator (Table 2). PP reflected both SBP and DBP while excluding redundancy and collinearity between these two pa- 


\begin{tabular}{|c|c|c|c|c|}
\hline \multirow[b]{3}{*}{ Parameters } & \multicolumn{4}{|c|}{ First PCA loading } \\
\hline & \multicolumn{2}{|l|}{ Male } & \multicolumn{2}{|l|}{ Female } \\
\hline & Age included & Age excluded & Age included & Age excluded \\
\hline Age (years) & 0.389 & - & 0.700 & - \\
\hline Waist circumference $(\mathrm{cm})$ & 0.666 & 0.688 & 0.681 & 0.692 \\
\hline Pulse pressure $(\mathrm{mmHg})$ & 0.321 & 0.227 & 0.535 & 0.482 \\
\hline Fasting blood sugar (mg/dL) & 0.489 & 0.403 & 0.519 & 0.526 \\
\hline Triglyceride $(\mathrm{mg} / \mathrm{dL})$ & 0.625 & 0.713 & 0.614 & 0.690 \\
\hline High-density lipoprotein $(\mathrm{mg} / \mathrm{dL})$ & -0.599 & -0.657 & -0.558 & -0.629 \\
\hline Eigenvalue & 1.685 & 1.627 & 2.198 & 1.859 \\
\hline Total variance (\%) & 28.08 & 32.54 & 36.63 & 37.18 \\
\hline
\end{tabular}

Table 3. First principle components of the parameters for metabolic syndrome. Pulse pressure $=$ systolic blood pressure - diastolic blood pressure. All parameters are $P<0.001$. PCA principal component analysis.

rameters with respect to the relationship with age. Therefore, although PP did not show the strongest correlation with age in females, PP was used as the blood pressure indicator in both sexes.

Principal component analysis. Based on the results of the correlation analysis, variables such as waist circumference (WC), PP, fasting blood sugar (FBS) level, TG level, and HDL-C level were selected as candidate biomarkers for the principal component analysis (PCA). First, factor analysis, including age, was performed to assess the association with age, and the principal components were found to have a significant positive correlation $(0.389$ in males, 0.700 in females) with age (Table 3 ). The proposed indicators, which correlated closely with age, were defined as principal components that reflected MS-BA. Second, we excluded age from the analysis to evaluate the influence of age and establish the correlation of the principal components with other biomarkers. This re-analysis confirmed the influence of age on the principal components. The principal components accounted for $32.54 \%$ of the total variance in males and $37.18 \%$ of that in females, with Eigenvalues of 1.627 and 1.859 , respectively (Table 3 ).

$B A$ algorithm and correction of BA estimation equation. Principal component scores derived after excluding age were used as an index of health status and BA in MS. Regression analysis was performed using five candidate MS biomarkers (WC, FBS, TG, HDL-C, and PP) as independent variables. The equations developed after the process for calculating the biological age score (BAS) were as follows:

BAS in males $=-6.333+0.055 \times(\mathrm{WC})+0.016 \times(\mathrm{PP})+0.019 \times(\mathrm{FBS})+0.006 \times(\mathrm{TG})-0.034 \times($ HDL-C $)$

BAS in females $=-5.917+0.043 \times(\mathrm{WC})+0.026 \times(\mathrm{PP})+0.023 \times(\mathrm{FBS})+0.007 \times(\mathrm{TG})-0.027 \times(\mathrm{HDL}-\mathrm{C})$

It is difficult to explain BA to the general public as it is not expressed in terms of years. To overcome this disadvantage, $\mathrm{BA}$ was converted into years using the T-scale considering that the scores are distributed with a mean of 0 and a standard deviation (SD) of 1.0 as follows:

$$
\begin{aligned}
\mathrm{BA} \text { in males } & =(\mathrm{BAS} \text { in males } \times 14.158)+48.779 \\
\mathrm{BA} \text { in females } & =(\mathrm{BAS} \text { in females } \times 14.261)+52.040
\end{aligned}
$$

With the abovementioned relationship between age and BA, BA can be underestimated at the upper range of the equation and overestimated at the lower range. To reduce this systemic error, we used the following correction equation:

$$
\begin{aligned}
& \text { Corrected } \mathrm{BA}=\mathrm{BA}+\mathrm{z} \\
& \mathrm{z}=(\mathrm{yi}-\mathrm{y}) \times(1-\mathrm{b})=(\text { age }- \text { mean age }) \times(1-\text { correlation with age and BA }) \\
& \text { Corrected BA in males }=-82.688+0.779 \times(\mathrm{WC})+0.227 \times(\mathrm{PP})+0.269 \times(\mathrm{FBS}) \\
& \quad+0.085 \times(\mathrm{TG})-0.481 \times(\mathrm{HDL}-\mathrm{C})+0.857 \times(\text { age }) \\
& \text { Corrected BA in females }=-60.340+0.613 \times(\mathrm{WC})+0.371 \times(\mathrm{PP})+0.328 \times(\mathrm{FBS}) \\
& \quad+0.100 \times(\mathrm{TG})-0.385 \times(\mathrm{HDL}-\mathrm{C})+0.538 \times(\text { age })
\end{aligned}
$$

The correlation coefficients between the corrected BA (cBA) and age calculated from the abovementioned equation were 0.711 and 0.748 for the males and females, respectively. Through this correction, under- and overestimations of BA were avoided (Fig. 1).

Influencing parameters of MS-BA. The relative impact of the risk factors on MS-BA is shown in Table 2. The scoring coefficients obtained from PCA were standardized values without units of measurement. Larger numbers indicated a greater influence on MS-BA. The most influential parameter in males was TG (0.43813), while it was WC in females (0.3722). 

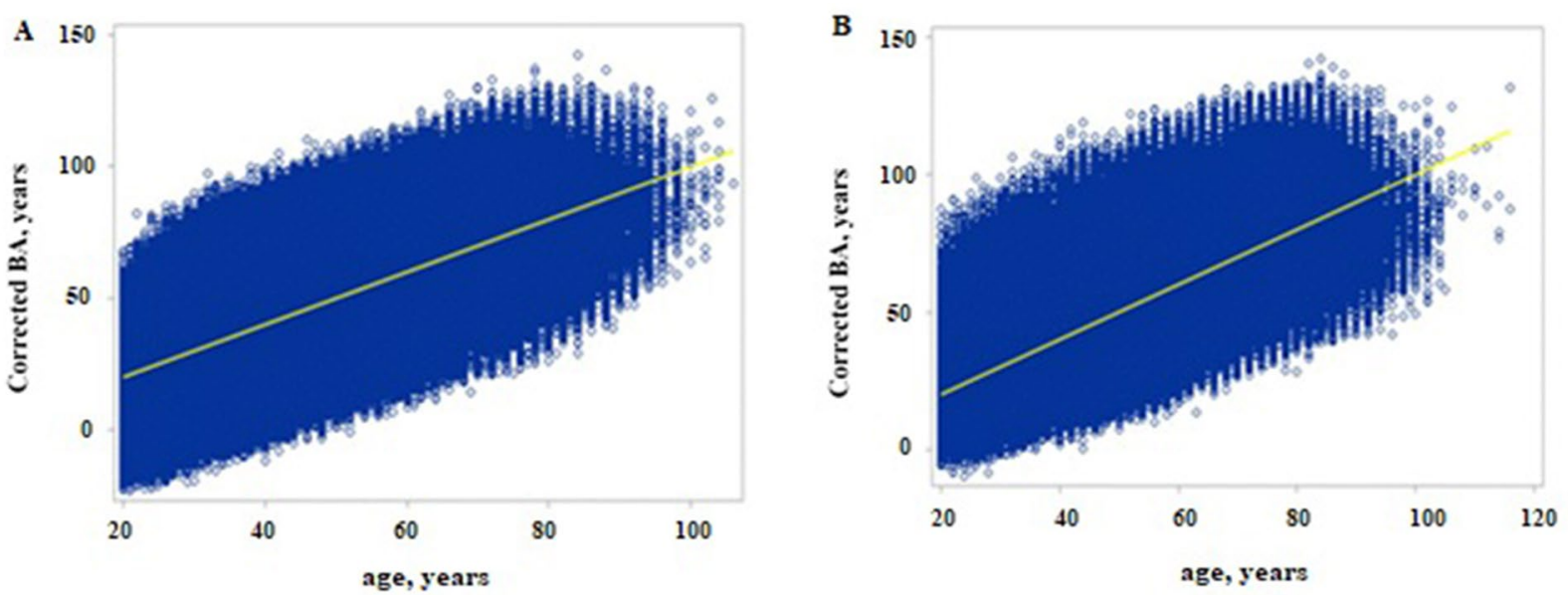

Figure 1. Relationship of MS BA in function of age for male (A), Female (B).

\begin{tabular}{|c|c|c|c|c|c|c|c|}
\hline \multirow[b]{2}{*}{ Age group } & \multicolumn{2}{|l|}{ Normal } & \multicolumn{2}{|l|}{ Risk } & \multicolumn{2}{|c|}{ Metabolic syndrome } & \multirow[b]{2}{*}{$P$ value } \\
\hline & N (\%) & Mean \pm SD & $\mathrm{N}(\%)$ & Mean \pm SD & $\mathbf{N}(\%)$ & Mean \pm SD & \\
\hline \multicolumn{8}{|l|}{ Male } \\
\hline TG $(\mathrm{n}=8,671,838)$ & $2,002,914(100)$ & $-12.64 \pm 8.86$ & $4,872,818(100)$ & $-0.78 \pm 10.78$ & $1,796,106(100)$ & $16.23 \pm 10.11$ & $<.0001$ \\
\hline YG $(\mathrm{n}=2,463,455)$ & $823,782(41.1)$ & $-11.76 \pm 8.66$ & $1,287,877(26.4)$ & $0.94 \pm 10.88$ & $351,796(19.6)$ & $19.18 \pm 9.97$ & $<.0001$ \\
\hline MG $(\mathrm{n}=4,118,565)$ & $857,424(42.8)$ & $-12.65 \pm 8.83$ & $2,338,587(48.0)$ & $-0.40 \pm 10.73$ & $922,554(51.4)$ & $16.45 \pm 10.07$ & $<.0001$ \\
\hline OG $(\mathrm{n}=2,089,818)$ & $321,708(16.1)$ & $-14.84 \pm 9.05$ & $1,246,354(25.6)$ & $-3.28 \pm 10.33$ & $521,756(29)$ & $13.84 \pm 9.68$ & $<.0001$ \\
\hline \multicolumn{8}{|l|}{ Female } \\
\hline TG $(n=7,846,694)$ & $2,677,507(100)$ & $-9.06 \pm 8.07$ & $3,889,567(100)$ & $0.77 \pm 9.71$ & $1,279,620(100)$ & $16.5 \pm 10.56$ & $<.0001$ \\
\hline YG $(n=1,376,061)$ & $840,140(31.4)$ & $-5.50 \pm 7.47$ & 480,365 (12.4) & $6.64 \pm 9.62$ & $55,556(4.3)$ & $27.64 \pm 9.94$ & $<.0001$ \\
\hline MG $(n=4,022,863)$ & $1,480,041(55.3)$ & $-9.85 \pm 7.58$ & $2,042,509(52.5)$ & $1.71 \pm 9.26$ & $500,313(39.1)$ & $19.57 \pm 9.82$ & $<.0001$ \\
\hline OG $(\mathrm{n}=2,447,770)$ & $357,326(13.3)$ & $-14.18 \pm 7.72$ & $1,366,693(35.1)$ & $-2.70 \pm 9.05$ & $723,751(56.6)$ & $13.53 \pm 9.90$ & $<.0001$ \\
\hline
\end{tabular}

Table 4. Clinical applications of the developed MS BA model. Young group: $20.0 \leq$ Age $<40.0$ years, Middle group: $40.0 \leq$ Age $<60.0$ years, Old group: Age $\geq 60.0$ years. $T G$ total group, $Y G$ young group, $M G$ middle group, $O G$ old group, $S D$ standard deviation.

Clinical applications of the developed MS-BA model. To evaluate the possibility of the clinical application of the MS-BA estimation model, three patient categories were created: normal group, risk group (with $1-2$ risk indicators), and MS group. Additionally, the subjects were divided three groups by chronological age as the young group ( $20-<40$ years), middle group ( $40-<60$ years), and old group ( $\geq 60$ years). Analysis of variance for the comparison of the mean differences between MS-BA and chronological age in the three groups showed significant differences $(P<0.001$; Table 4$)$. It should be noted that a positive difference between MS-BA and chronological age indicated a high risk, whereas a negative difference indicated a low risk.

Limitations of conventional MS diagnosis assessed using MS-BA. In $6.49 \%$ of males and $12.9 \%$ of females who were assigned to the normal group based on conventional MS diagnosis, MS-BA was higher than chronological age (i.e., the difference exceeded zero). These participants, thus, appeared normal based on conventional diagnostic criteria, but were not normal when assessed using MS-BA. In contrast, $4.81 \%$ of males and $4.49 \%$ of females who were assigned to the MS group based on conventional diagnostic criteria had MS-BA lower than the chronological age (i.e., the difference was less than zero). These participants were conventionally diagnosed as having MS but were actually normal. If MS-BA were applied in parallel with the conventional diagnosis of MS, the MS status could be effectively identified and managed (Table 5; Fig. 2).

Relationships between lifestyle factors and MS-BA. Smoking. Lifestyle-related biomarkers were selected based on the self-report questionnaire on health behavior administered during NHIS health screening examinations. Smoking status was divided into three categories: never smoker, former smoker, and current smoker. Smoking as a continuous variable was calculated as follows: pack/day $\times$ year. The mean and standard deviation of the differences between MS-BA and chronological age (cBA-age) were calculated according to the smoking categories. In both sexes, the differences in values increased as the amount of packs/day in- 


\begin{tabular}{|c|c|c|c|c|c|c|}
\hline \multirow[b]{3}{*}{ Conventional methods } & \multicolumn{6}{|l|}{ cBA-age } \\
\hline & \multicolumn{3}{|l|}{ Male } & \multicolumn{3}{|l|}{ Female } \\
\hline & $\begin{array}{l}\text { Over zero } \\
\text { N (\%) }\end{array}$ & $\begin{array}{l}\text { Under zero } \\
\text { N (\%) }\end{array}$ & Total & $\begin{array}{l}\text { Over zero } \\
\text { N (\%) }\end{array}$ & $\begin{array}{l}\text { Under zero } \\
\mathrm{N}(\%)\end{array}$ & Total \\
\hline Normal & $130,029(6.49 \%)$ & $1,872,885(93.51 \%)$ & $2,002,914$ & $345,460(12.9 \%)$ & $2,332,047(87.1 \%)$ & $2,677,507$ \\
\hline Risk & $2,378,695(48.82 \%)$ & $2,494,123(51.18 \%)$ & $4,872,818$ & $2,059,727(52.96 \%)$ & $1,829,840(47.04 \%)$ & $3,889,567$ \\
\hline Metabolic syndrome & $1,709,784(95.19 \%)$ & $86,322(4.81 \%)$ & $1,796,106$ & $1,222,160(95.51 \%)$ & $57,460(4.49 \%)$ & $1,279,620$ \\
\hline Total & $4,218,508(48.65 \%)$ & $4,453,330(51.35 \%)$ & 8671,838 & $3,627,347(46.23 \%)$ & $4,219,347(53.77 \%)$ & $7,846,694$ \\
\hline
\end{tabular}

Table 5. The discrepancy between conventional diagnosis of metabolic syndrome and MS BA. All parameters are $P<0.001$.
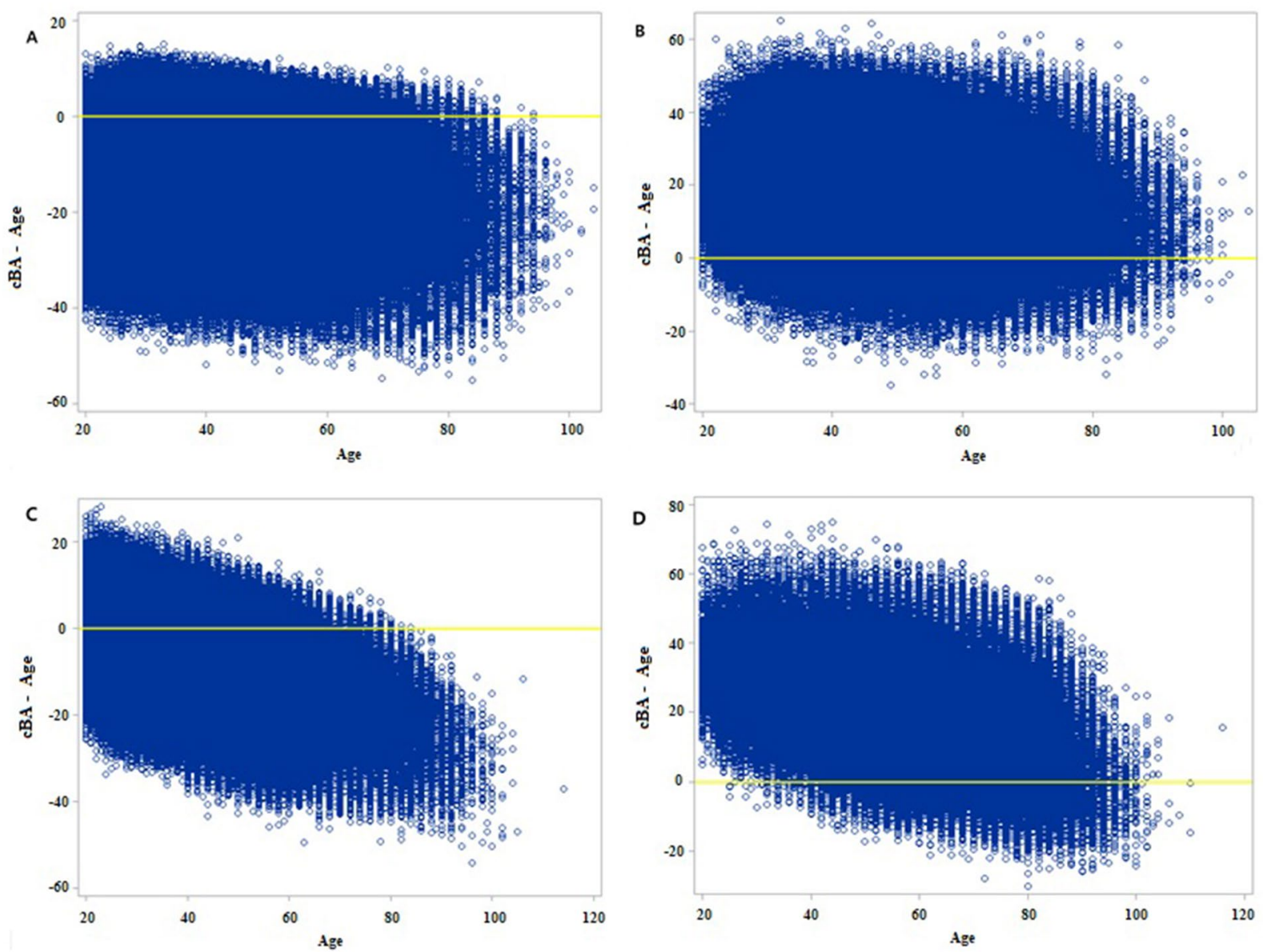

Figure 2. The scatter plot of discrepancy between conventional diagnosis. Y axis means the difference of MS BA and chronological age. $\mathrm{X}$ axis was chronological age. (A) normal male group, (B) MS male group, (C) normal female group, (D) MS female group.

creased, indicating that smoking could increase MS-BA (Table 6). Smoking as a continuous variable was then included as an independent variable in the linear regression analysis. The equations were as follows:

$$
\begin{gathered}
\text { cBA-age in males }=-0.8763+0.08062 \times(\text { pack } \times \text { year }), P<0.00 \\
\text { cBA-age in females }=-0.0668+0.14135 \times(\text { pack } \times \text { year }), P<0.001
\end{gathered}
$$

The scatterplots are shown in Fig. 3. The results showed that the higher the amount of smoking, the worse was the MS-BA in both males and females.

Alcohol consumption. The daily alcohol consumption was calculated as the drinking amount (ml)/day $\times$ alcohol content $(\%) \times 0.8$ (alcohol gravity)/100, following the International Guide for Monitoring Alcohol Consumption and Related Harm of the World Health Organization (WHO). Alcohol consumption was categorized into four as abstinent, low risk, medium risk, and high risk. The mean and standard deviation of the differences between MS-BA and chronological age (cBA-Age) were calculated according to the alcohol consumption categories. We 


\begin{tabular}{|l|l|c|c|c|c|}
\hline \multirow{4}{*}{ Lifestyles } & \multicolumn{5}{|l|}{ cBA-age } \\
\cline { 3 - 6 } & \multirow{4}{|l|}{ Male } & \multicolumn{2}{l|}{ Female } \\
\cline { 2 - 6 } & Categories & $\mathbf{N}(\%)$ & Mean \pm SD & N (\%) & Mean \pm SD \\
\hline \multirow{4}{*}{ Smoking } & Never smoker & $2,734,418(31.5 \%)$ & $-1.304 \pm 13.604$ & $7,413,754(94.5 \%)$ & $-0.109 \pm 12.775$ \\
\cline { 2 - 6 } & Former smoker & $2,641,443(30.5 \%)$ & $0.441 \pm 13.662$ & $163,979(2.1 \%)$ & $0.868 \pm 13.228$ \\
\cline { 2 - 6 } & Current smoker & $3,293,387(38.0 \%)$ & $0.737 \pm 14.542$ & $265,405(3.4 \%)$ & $1.945 \pm 13.709$ \\
\hline \multirow{4}{*}{ Alcohol consumption } & Abstinent & $701,381(10.9 \%)$ & $-0.605 \pm 13.756$ & $1,216,374(37.4 \%)$ & $-0.293 \pm 12.587$ \\
\cline { 2 - 6 } & Low risk & $5,125,490(79.5 \%)$ & $-0.0002 \pm 14.038$ & $1,845,045(56.7 \%)$ & $-0.855 \pm 12.144$ \\
\cline { 2 - 6 } & Medium risk & $425,377(6.6 \%)$ & $1.067 \pm 14.650$ & $144,624(4.4 \%)$ & $1.340 \pm 12.494$ \\
\cline { 2 - 6 } & High risk & $193,821(3.0 \%)$ & $1.906 \pm 14.918$ & $48,557(1.5 \%)$ & $2.377 \pm 13.113$ \\
\hline \multirow{3}{*}{ Physical activity } & $<600 \mathrm{METs}$ & $2,569,888(29.6 \%)$ & $0.654 \pm 14.228$ & $3,069,129(39.1 \%)$ & $0.571 \pm 12.795$ \\
\cline { 2 - 6 } & $\geq 600,<3000 \mathrm{METs}$ & $4,986,903(57.5 \%)$ & $0.023 \pm 13.961$ & $4,069,269(51.9 \%)$ & $-0.282 \pm 12.530$ \\
\cline { 2 - 6 } & $\geq 3000 \mathrm{METs}$ & $1,115,047(12.9 \%)$ & $-1.591 \pm 13.619$ & $708,296(9.0 \%)$ & $-1.073 \pm 12.494$ \\
\hline
\end{tabular}

Table 6. The relationship between lifestyles and MS BA. All parameters are $P<0.001$.

found that the greater the amount of alcohol consumed, the worse was the MS-BA in males. Similar tendencies were obtained among females, except in the low-risk group (Table 6). The daily alcohol consumption biomarker was included as an independent variable in the linear regression analysis. The equations were as follows:

$$
\begin{aligned}
\mathrm{cBA} \text {-age in males } & =-0.53628+0.03701 \times(1 \text { day alcohol amount }), \mathrm{P}<0.001 \\
\mathrm{cBA} \text {-age in females } & =-0.50761+0.04419 \times(1 \text { day alcohol amount }), \mathrm{P}<0.001
\end{aligned}
$$

The scatterplots are shown in Fig. 3. The results showed that the greater the amount of alcohol consumed, the worse was the MS-BA in both males and females.

Physical activity. Physical activity was defined the sum of the physical activity levels for 1 week based on the WHO International Physical Activity Questionnaire (IPAQ) standard, according to which physical activity was divided into three categories: low level (<600 MET*MIN), medium level (600-3000 MET*MIN), and high level $\left(>3000 \mathrm{MET}^{\star} \mathrm{MIN}\right)$. The mean and standard deviation of the differences between MS-BA and chronological age (cBA-Age) were calculated for each of the physical activity categories. The more the physical activity, the greater the difference in MS-BA and chronological age in both males and females. This indicated that as the physical activity increased, the MS-BA decreased (Table 6).

The sum of 1 week of physical activity as a continuous variable was included as an independent variable in the linear regression analysis. The equations were as follows:

$$
\begin{aligned}
\text { cBA-Age in Males } & =0.78723-0.0005245 \times(1-\text { week physical activity }), \mathrm{P}<0.001 \\
\text { cBA-Age in Females } & =0.47183-0.0004039 \times(1-\text { week physical activity }), \mathrm{P}<0.001
\end{aligned}
$$

The scatterplots are shown in Fig. 3. The results showed that the more the physical activity, the lesser the MS-BA in both males and females.

Multiple regression analysis of lifestyle factors and MS-BA. The amounts of smoking, daily alcohol consumption, and 1 week's physical activity as continuous data were included as independent variables in the multiple linear regression analysis. The equations were as follows:

$$
\begin{aligned}
\text { cBA-age in males }= & -0.419+0.025 \times(1 \text { day's alcohol amount })-0.000512 \times(1 \text { week's physical activity }) \\
& +0.076 \times(\text { smoking amount }), \mathrm{P}<0.001 \\
\mathrm{cBA} \text {-age in females }= & -0.025+0.037 \times(1 \text { day's alcohol amount })-0.0004123 \times(1 \text { week's physical activity }) \\
& +0.132 \times(\text { smoking amount }), \mathrm{P}<0.001
\end{aligned}
$$

Compared with the results of the simple linear regression analysis, those of the multiple regression analysis showed that each of these independent variables had similar values and directions. As the smoking and drinking increased and physical activity decreased, the MS-BA increased in both males and females. In addition, the amount of smoking and drinking had a more profound impact in females more than males, whereas the amount of physical activity had greater effects in males than in females. These results allowed us to quantify the influence of smoking, drinking, and physical activity on MS-BA.

\section{Discussion}

In this nationally representative longitudinal study, we demonstrated that an MS-BA model may be used as a supplementary tool to the conventional MS diagnostic criteria to more accurately evaluate and manage MS. Moreover, we found that in both sexes, the MS-BAs decreased as the amount of smoking and drinking increased 

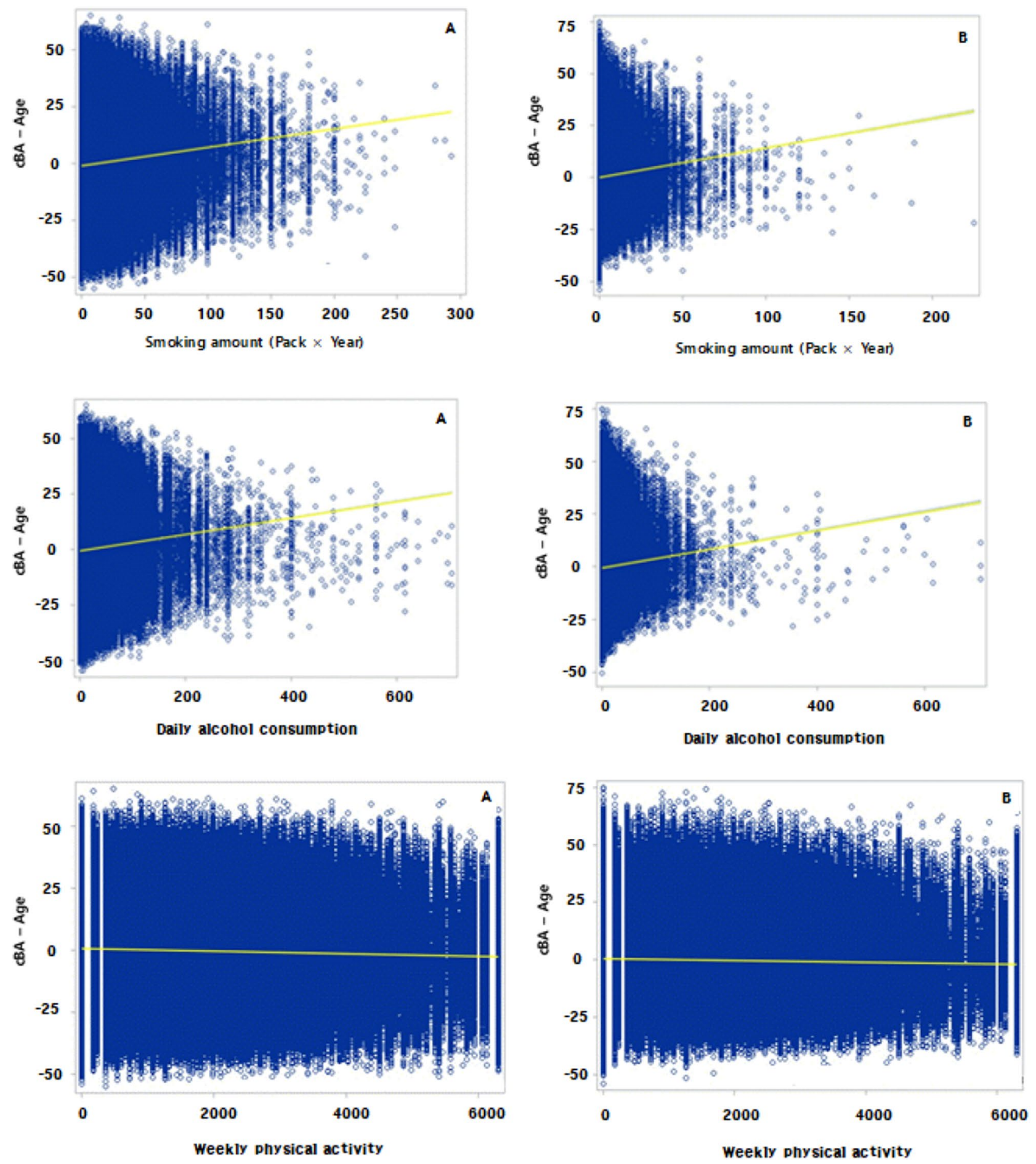

Figure 3. Relationships between lifestyle factors and MS BA (A: Male, B: Female).

and the amount of physical activity decreased. To the best of our knowledge, this is the first study to quantitatively measure the effects of lifestyle on the MS-BA in a large population.

Aging is characterized by a time-related decline in physiological functions, and the rate of aging differ among individuals owing to the variability of disease ${ }^{23}$. However, chronological age is simply explained by the flow of time and has limitations when used to evaluate an individuals' physiological function, health, and aging status ${ }^{24}$. The concept of BA has been widely investigated since the 1970s, and it has been proposed to quantify and digitize the aging state based on the age-related characteristic changes in physical and physiological functions ${ }^{25}$. There has been a growing interest in utilizing BA in chronic health management to compensate for the limitations of the binary structure of disease diagnosis ${ }^{26-28}$. BA is generally derived from a combination of biomarkers and represents an individual's overall health and aging state in comparison with those of the same sex and age ${ }^{25,29}$. 
It has previously been reported that BA can be easily used as a continuous index to monitor health and aging status and can also be communicated easily to healthcare consumers ${ }^{30-32}$.

MS is diagnosed when three or more of the five parameters are outside of the reference range. However, the conventional diagnostic method has limitations in that it cannot reflect the current status of MS correctly with respect to an individual's age and sex. For example, during the management of MS, some parameters may be well-controlled, while others could worsen. Thus, there is some difficulty in assessing whether the status of MS has really improved after lifestyle modification.

Numerous studies have assessed the effects of changes in MS components, such as serum glucose level, cholesterol level, blood pressure, and WC. The elevation of each of these components has been established to be associated with a higher MS risk. However, few studies have considered the individual association of these factors with MS-BA. In this study, WC, TG levels, and HDL-C level had high impact scores on MS-BA, and these scores were higher for males than for females. Meanwhile, PP and FBS had relatively lower scores than the other parameters, and the scores were lower in males than in females. Using the MS-BA index, which is a continuous variable, enables a closer assessment of the relationship between these parameters and MS. Further, their effects on the normal aging process, disease progression, and intervention outcome are measured and evaluated more objectively. In healthcare, indicators of outcome measures should be more relevant and sensitive. In this context, MS-BA can be utilized as a novel evaluation and management index in the assessment of the overall state of MS.

The present study demonstrated methods of quantitatively measuring the effects of lifestyle factors, such as smoking, drinking, and physical activity, on MS-BA. As continuous variables, we calculated the amount of smoking per year, alcohol consumption per day, and physical activity per week from the self-reported questionnaire on health behavior administered during the NHIS health screening examinations. We constructed linear regression equations with lifestyle behaviors and MS-BA. Therefore, our approach makes it possible to quantitatively measure the effects of changes in lifestyle behavior on MS-BA.

Our results support those of previous studies that found an influence of smoking on the development of visible signs of aging ${ }^{20}$. Smoking may increase sympathetic activity and circulating cortisol, catecholamine, vasopressin, and growth hormone levels. Therefore, it has been considered to play a causal role in the development of $\mathrm{MS}^{33}$. Additionally, current smokers, particularly those with excessive consumption, have a higher risk of $\mathrm{MS}^{34,35}$. Moreover, the relationship of smoking and alcohol consumption with the development of MS was found to be sex specific $^{36}$. Similarly, we found that smoking increased the MS-BA and that the amount of smoking had a more profound impact on females than on males.

A previous meta-analysis showed that heavy alcohol consumption might be associated with a higher risk of MS; accordingly, very light alcohol consumption seemed to be associated with a lower risk of $\mathrm{MS}^{37}$. Further, this risk was greater in men than in women. In contrast, we found that alcohol consumption had a greater impact on the risk of MS in females than in males. Regular physical activity can yield physiological improvements that in turn reduces the rate of aging. A systematic review showed that the BA increased with decreased physical activity, irrespective of the $\operatorname{sex}^{38}$. Further, active middle-aged men who followed a regular endurance exercise program have a, on average, 4.7 years younger BA than their chronological ages ${ }^{39}$. However, it should be noted that an adequate amount of exercise is required to obtain the beneficial effects of exercise on cardiorespiratory function ${ }^{40}$. In agreement with these findings, we found that subjects with a high level of physical activity $(>3000$ $\mathrm{MET}^{\star} \mathrm{MIN}$ ) had the lowest value of cBA-age in both sexes, which indicated a younger MS-BA. Physical activity could exert its protective effects against MS by improving plasma lipid levels, particularly through increases in HDL-C levels and decreases in TG levels. In addition, physical activity has been shown to lower blood pressure, improve glucose tolerance and insulin sensitivity, and lower the risk of type 2 diabetes $^{19}$.

In conclusion, the MS-BA model accurately reflects the MS status relative to the individual's age and sex. Thus, it can be a supplementary tool for evaluating and managing MS, quantitatively measuring the effect of lifestyle changes on MS, and motivating patients to maintain a healthy lifestyle. We hope that the estimation of MS-BA can facilitate the evaluation of the influence of age and sex on the MS status.

\section{Methods}

Study design and population. MS involves a clustering of abdominal obesity, elevated blood pressure, low serum HDL-C levels, elevated serum TG levels, and impaired FBS ${ }^{41}$. The modified NCEP-ATP III diagnostic criteria for $\mathrm{MS}^{42}$, developed by the American Heart Association/National Heart, Lung, and Blood Institute, stipulates that MS diagnosis requires meeting three of the following five criteria: central obesity (WC: males $\geq 90 \mathrm{~cm}$, females $\geq 85 \mathrm{~cm}$ ), high blood pressure (SBP/DBP $\geq 130 / 85 \mathrm{mmHg}$ or medication intake), high TG levels ( $\geq 150 \mathrm{mg} / \mathrm{dL}$ or medication intake), low HDL-C levels (males $<40 \mathrm{mg} / \mathrm{dL}$, females $<50 \mathrm{mg} / \mathrm{dL}$, or medication intake), and fasting hyperglycemia $(\geq 100 \mathrm{mg} / \mathrm{dL} \text { or medication intake })^{43}$.

This study used the Medical and Health Examination database of the National Health Insurance ServiceHealth Screening Cohort (NHIS-HEALS). Data of 21,317,002 subjects who participated in the NHIS health screening examinations from 2014 to 2015 were collected from the NHIS-HEALS. Among them, 4,800,000 subjects were excluded due to the inclusion criteria listed in the Table 1.

This study was approved and exempted from review by the institutional review board of the NHIS Ilsan Hospital (NHIMC 2018-01-009) owing to the use of anonymized data.

Data source. The NHIS of Korea was launched as a single insurance system and adopted as a compulsory social insurance program covering the whole population living in the country. Furthermore, the NHIS provides biannual health screening examinations for all citizens aged $\geq 40$ years. These include a self-report questionnaire on health behavior; measurements of height, weight, and blood pressure; and urine and blood test results. The NHIS-HEALS provides cohort data of participants who undergo health screening examinations for 
research purposes ${ }^{44}$. It has been used in several epidemiological studies, and its validity is described in detail elsewhere $e^{45,46}$.

Measures and definitions. Six biomarkers were selected: WC, SBP, and DBP as physical measures and FBS, TG, and HDL-C levels as blood test parameters. Additionally, lifestyle-related biomarkers were selected using the self-reported questionnaire on health behavior. Smoking was divided into three categories: never smoker, former smoker, and current smoker. Smoking amount as a continuous variable was calculated as follows: pack/day $\times$ year. Daily alcohol consumption was calculated as the drinking amount $(\mathrm{ml}) /$ day $\times$ alcohol content $(\%) \times 0.8$ (alcohol gravity)/100 based on the International Guide for Monitoring Alcohol Consumption and Related Harm. It was divided into four categories: abstinent, low risk, medium risk, and high risk. Physical activity was defined as the sum of physical activity levels for 1 week based on the WHO IPAQ standard. It was divided into three categories: low level (<600 MET`MIN), medium level (600-3000 MET^MIN), and high level $\left(>3000 \mathrm{MET}^{\star} \mathrm{MIN}\right)^{47}$.

Statistical analysis. Correlation analysis and assessment of redundancy. MS diagnostic parameters, namely, WC, SBP, DBP, FBS level, TG levels, and HDL-C level, were used to develop the MS-BA model. First, linear correlation analysis of age and measured parameters was performed. Redundancy of the parameters was suspected based on the high level of correlation observed between the individual parameters in the correlation analysis. The correlation with age was assessed after calculating MBP and PP based on SBP and DBP to avoid redundancy, and both $\mathrm{MBP}$ and $\mathrm{PP}$ parameters were then used in model development ${ }^{26}$.

Principal component analysis. PCA was used to estimate MS-BA. First, PCA was performed using age and the five MS diagnostic parameters as variables. Among these factors, the factor with the highest Eigenvalue, which is the sum of the total variance of the parameter, was considered the principal component. We confirmed the changes in the factor loading value, which indicated the correlation between the factors and variables of PCA, after the exclusion of age.

Construction of BA. BAS was developed using the first principal component obtained from PCA of the selected biomarkers. Individual BAS was transformed into terms of years (BA) using the T-scale (transformation from a standard score to T-score) with consideration that these scores were distributed with a mean of 0 and an SD of 1.0. The formula for converting BAS to BA is as follows:

$$
\mathrm{BA}=(\mathrm{BAS} \times \text { standard deviation of } \mathrm{CA})+\text { mean of } \mathrm{CA} .
$$

Correction of BA estimation equation. Calculation of BA using the abovementioned formula underestimated the means for BA at the upper end of the regression and overestimated it at the lower end. To correct this systemic error, $\mathrm{cBA}$ was calculated using the following correction method:

$$
\text { Corrected } \mathrm{BA}=\mathrm{BA}+\mathrm{z} \text {. }
$$

The $\mathrm{z}$ value is as follows. $\mathrm{z}=\left(\mathrm{y}_{\mathrm{i}}-\mathrm{y}\right) \times(1-b)$, where " $\mathrm{y}_{\mathrm{i}}$ " is the chronological age of an individual, " $\mathrm{y}$ " is the average chronological age of all samples, and $b$ is the coefficient of simple linear regression, which expresses the relationship between BA and chronological age.

Multiple linear regression analysis between lifestyle factors and MS-BA. Multiple regression analysis was used to identify the relationship between age and lifestyle factors such as smoking, drinking, and physical activity.

All statistical analyses were performed using SAS version 9.4 (SAS Institute, Cary, NC, USA. https://www.sas. com/en_us/home.html). A two-sided $P$ value of $<0.05$ was considered statistically significant.

\section{Data availability}

The datasets generated during the current study are available from the corresponding author on reasonable request.

Received: 14 May 2020; Accepted: 2 December 2020

Published online: 11 January 2021

\section{References}

1. World Health Organization. World Health Statistics 2014: A Wealth of Information on Global Public Health (World Health Organization, 2014) https://apps.who.int/iris/handle/10665/112739.

2. Lee, S. H., Tao, S. \& Kim, H. S. The prevalence of metabolic syndrome and its related risk complications among Koreans. Nutrients 11(8), 1755 (2019).

3. Lim, S. et al. Components of metabolic syndrome in Korean adults: A hospital-based cohort at Seoul National University Bundang Hospital. J. Obes. Metab. Syndr. 28(2), 118-128 (2019).

4. VanWormer, J. J., Boucher, J. L., Sidebottom, A. C., Sillah, A. \& Knickelbine, T. Lifestyle changes and prevention of metabolic syndrome in the Heart of New Ulm Project. Prev. Med. Rep. 6, 242-245 (2017).

5. Kassi, E., Pervanidou, P., Kaltsas, G. \& Chrousos, G. Metabolic syndrome: Definitions and controversies. BMC Med. 9, 48 (2011).

6. Huang, P. L. A comprehensive definition for metabolic syndrome. Dis. Models Mech. 2(5-6), 231-237 (2009).

7. Goodman, E., Daniels, S. R., Meigs, J. B. \& Dolan, L. M. Instability in the diagnosis of metabolic syndrome in adolescents. Circulation 115(17), 2316-2322 (2007). 
8. Patni, R. \& Mahajan, A. The metabolic syndrome and menopause. J. Midlife Health 9(3), 111-112 (2018).

9. Kwon, H. S. et al. Prevalence and clinical characteristics of the metabolic syndrome in middle-aged Korean adults. Korean J. Intern. Med. 20(4), 310-316 (2005).

10. Ford, E. S., Giles, W. H. \& Dietz, W. H. Prevalence of the metabolic syndrome among US adults: Findings from the third National Health and Nutrition Examination Survey. JAMA 287(3), 356-359 (2002).

11. Park, H. S., Lee, S. Y., Kim, S. M., Han, J. H. \& Kim, D. J. Prevalence of the metabolic syndrome among Korean adults according to the criteria of the International Diabetes Federation. Diabetes Care 29(4), 933-934 (2006).

12. Ford, E. S., Giles, W. H. \& Mokdad, A. H. Increasing prevalence of the metabolic syndrome among US adults. Diabetes Care 27(10), 2444-3244 (2004).

13. Karvonen-Gutierrez, C. \& Kim, C. Association of Mid-Life Changes in Body Size, Body Composition and Obesity Status with the Menopausal Transition. Healthcare (Basel). 4(3), 42 (2016).

14. Laaksonen, D. E. et al. Testosterone and sex hormone-binding globulin predict the metabolic syndrome and diabetes in middleaged men. Diabetes Care 27(5), 1036-1041 (2004).

15. Ingram, D. K., Nakamura, E., Smucny, D., Roth, G. S. \& Lane, M. A. Strategy for identifying biomarkers of aging in long-lived species. Exp. Gerontol. 36(7), 1025-1034 (2001).

16. Pouliot, M. C. et al. Waist circumference and abdominal sagittal diameter: Best simple anthropometric indexes of abdominal visceral adipose tissue accumulation and related cardiovascular risk in men and women. Am. J. Cardiol. 73(7), 460-468 (1994).

17. Mitnitski, A. B., Graham, J. E., Mogilner, A. J. \& Rockwood, K. Frailty, fitness and late-life mortality in relation to chronological and biological age. BMC Geriatr. 2(1), 1-8 (2002).

18. Isomaa, B. et al. Cardiovascular morbidity and mortality associated with the metabolic syndrome. Diabetes Care 24(4), 683-689 (2001).

19. Zhu, S., St-Onge, M. P., Heshka, S. \& Heymsfield, S. B. Lifestyle behaviors associated with lower risk of having the metabolic syndrome. Metabolism 53(11), 503-1511 (2004).

20. Schou, A. L., Mølbak, M. L., Schnor, P., Grønbæk, M. \& Tolstrup, J. S. Alcohol consumption, smoking and development of visible age-related signs: A prospective cohort study. J. Epidemiol. Commun. Health 71(12), 1177-1184 (2017).

21. Misra, A. \& Khurana, L. Obesity and the metabolic syndrome in developing countries. J. Clin. Endocrinol. Metab. 93(11 Suppl 1), S9-S30 (2008).

22. Galassi, A., Reynolds, K. \& He, J. Metabolic syndrome and risk of cardiovascular disease: A meta-analysis. Am. J. Med. 119(10), 812-819 (2006).

23. Anstey, K. J., Lord, S. R. \& Smith, G. A. Measuring human functional age: A review of empirical findings. Exp. Aging Res. 22(3), 245-266 (1996).

24. Borkan, G. A. \& Norris, A. H. Assessment of biological age using a profile of physical parameters. J. Gerontol. 35(2), 177-184 (1980).

25. Borkan, G. A. \& Norris, A. H. Biological age in adulthood: Comparison of active and inactive U.S. males. Hum Biol. 52(4), 787-802 (1980).

26. Kang, Y. G. et al. Models for estimating the metabolic syndrome biological age as the new index for evaluation and management of metabolic syndrome. Clin. Interv. Aging 12, 253-261 (2017).

27. Bae, C. Y. et al. A model for estimating body shape biological age based on clinical parameters associated with body composition. Clin. Interv. Aging 8, 11-18 (2013).

28. Bae, C. Y. et al. Models for estimating the biological age of five organs using clinical biomarkers that are commonly measured in clinical practice settings. Maturitas 75(3), 253-260 (2013).

29. Bae, C. Y. et al. Development of models for predicting biological age (BA) with physical, biochemical, and hormonal parameters. Arch. Gerontol. Geriatr. 47(2), 253-265 (2008).

30. Iguchi, A., Shimokata, H. \& Endo, H. Assessment of biological age and indices of aging. Nihon Ronen Igakkai Zasshi 33(11), 806-810 (1996).

31. Simpkin, A. J. et al. Are objective measures of physical capability related to accelerated epigenetic age? Findings from a British birth cohort. BMJ Open 7(10), e016708 (2017).

32. Facchini, F., Gueresi, P. \& Pettener, D. Biological age in Italian adults: Influence of social and behavioural factors. Ann. Hum. Biol. 19(4), 403-420 (1992).

33. Lei, M. K., Beach, S. R., Dogan, M. V. \& Philibert, R. A. A pilot investigation of the impact of smoking cessation on biological age. Am. J. Addict. 26(2), 129-135 (2017).

34. Balhara, Y. P. Tobacco and metabolic syndrome. Indian J. Endocrinol. Metab. 16(1), 81-87 (2012).

35. Chiolero, A., Faeh, D., Paccaud, F. \& Cornuz, J. Consequences of smoking for body weight, body fat distribution, and insulin resistance. Am. J. Clin. Nutr. 87(4), 801-809 (2008).

36. Huang, J. H. et al. Lifestyle factors and metabolic syndrome among workers: The role of interactions between smoking and alcohol to nutrition and exercise. Int. J. Environ. Res. Public Health 12(12), 15967-15978 (2015).

37. Sun, K. et al. Alcohol consumption and risk of metabolic syndrome: A meta-analysis of prospective studies. Clin. Nutr. 33(4), 596-602 (2014).

38. Bacil, E. D., Mazzardo Júnior, O., Rech, C. R., Legnani, R. F. \& de Campos, W. Physical activity and biological maturation: A systematic review. Rev. Paul. Pediatr. 33(1), 114-121 (2015).

39. Nakamura, E., Moritani, T. \& Kanetaka, A. Biological age versus physical fitness age. Eur. J. Appl. Physiol. Occup. Physiol. 58(7), 778-785 (1989).

40. Dubina, T. L., Mints, A. Y. \& Zhuk, E. V. Biological age and its estimation. III. Introduction of a correction to the multiple regression model of biological age in cross-sectional and longitudinal studies. Exp. Gerontol. 19(2), 133-143 (1984).

41. Alexander, C. M., Landsman, P. B., Teutsch, S. M. \& Haffner, S. M. NCEP-defined metabolic syndrome, diabetes, and prevalence of coronary heart disease among NHANES III participants age 50 years and older. Diabetes 52(5), 1210-1214 (2003).

42. Cleeman, J. I., Grundy, S. M., Becker, D. \& Clark, L. Expert panel on detection, evaluation, and treatment of high blood cholesterol in adults. Executive summary of the third report of The National Cholesterol Education Program (NCEP) expert panel on detection, evaluation, and treatment of high blood cholesterol in adults (adult treatment panel III). JAMA 285(19), 2486-2497 (2001).

43. Grundy, S. M. et al. Diagnosis and management of the metabolic syndrome: An American Heart Association/National Heart, Lung, and Blood Institute scientific statement. Circulation 12, 2735-2752 (2005).

44. Lee, J., Lee, J. S., Park, S. H., Shin, S. A. \& Kim, K. Cohort profile: The National Health Insurance Service-National Sample Cohort (NHIS-NSC), South Korea. Int. J. Epidemiol. 46(2), e15 (2017).

45. Seong, C. et al. Data resource profile: The national health information database of the National Health Insurance Service in South Korea. Int. J. Epidemiol. 46(3), 799-800 (2017).

46. Bae, Y. S. et al. Association of concurrent changes in metabolic health and weight on cardiovascular disease risk: A nationally representative cohort study. J. Am. Heart Assoc. 8(17), e011825 (2019).

47. Hallal, P. C. \& Victora, C. G. Reliability and validity of the international physical activity questionnaire (IPAQ). Med. Sci. Sports Exerc. 36(3), 556 (2004). 


\section{Acknowledgements}

This study was funded by the National Health Insurance Service and used NHIS-NSC data.

\section{Author contributions}

C.Y.B. conceptualized the study, designed the research, and conducted the big data analysis. M.P. interpreted the results and drafted the manuscript. D.K., K.H.C., and J.C. collected the data from the NHIS-HEALS database. M.P., Y.I., and S.K. critically revised the article for important intellectual content. All authors reviewed and contributed to the preparation of the final manuscript.

\section{Competing interests}

The authors declare no competing interests.

\section{Additional information}

Correspondence and requests for materials should be addressed to M.P.

Reprints and permissions information is available at www.nature.com/reprints.

Publisher's note Springer Nature remains neutral with regard to jurisdictional claims in published maps and institutional affiliations.

(c) Open Access This article is licensed under a Creative Commons Attribution 4.0 International License, which permits use, sharing, adaptation, distribution and reproduction in any medium or format, as long as you give appropriate credit to the original author(s) and the source, provide a link to the Creative Commons licence, and indicate if changes were made. The images or other third party material in this article are included in the article's Creative Commons licence, unless indicated otherwise in a credit line to the material. If material is not included in the article's Creative Commons licence and your intended use is not permitted by statutory regulation or exceeds the permitted use, you will need to obtain permission directly from the copyright holder. To view a copy of this licence, visit http://creativecommons.org/licenses/by/4.0/.

(C) The Author(s) 2021 